\title{
Near-field studies of anisotropic variations and temperature-induced structural changes in a supported single lipid bilayer
}

\author{
Merrell A. Johnson $\oplus^{1,2, *}$ and Ricardo S. Decca ${ }^{1, \dagger}$ \\ ${ }^{1}$ Department of Physics, Indiana University - Purdue University Indianapolis, 402 North Blackford Street, \\ Building LD154, Indianapolis, Indiana 46202, USA \\ ${ }^{2}$ Department of Physics, Purdue University Fort Wayne, 2101 E. Coliseum Blvd., Building KT126A, Fort Wayne, Indiana 46805, USA
}

(Received 20 November 2019; accepted 15 February 2020; published 27 March 2020)

\begin{abstract}
Temperature-controlled polarization modulation near-field scanning optical microscopy measurements of a single supported $L_{\beta^{\prime}}$ 1,2-dipalmitoyl-sn-glycero-3-phosphocholine (DPPC) lipid bilayer are presented. The effective retardance $\left(S=\frac{2 \pi\left(n_{e}-n_{o}\right) t}{\lambda}\right.$, where $t$ is the thickness of the bilayer and $\lambda$ is the wavelength of light used) and the direction of the projection of the acyl chains $(\theta)$ were measured simultaneously. We demonstrate how one is able to align the system over the sample and measure a relative retardance $\Delta S$, a crucial step in performing temperature-controlled experiments. Maps of $\Delta S$ and $\theta$, with a lateral resolution on the order of $\sim 100 \mathrm{~nm}$ are presented, highlighting variations deriving from changes in the average molecular orientation across a lipid membrane at room temperature. A discussion of how this information can be used to map the average three-dimensional orientation of the molecules is presented. From $\Delta S$ and the known thickness of the membrane $t$ the birefringence $\left(n_{e}-n_{o}\right)$ of the bilayer was determined. Temperature-controlled measurements yielded a change of $\Delta S \sim(3.8 \pm 0.3) \mathrm{mrad}$ at the main transition temperature $\left(T_{m} \sim 41^{\circ} \mathrm{C}\right)$ of a single planar bilayer of DPPC, where the membrane transitioned between the gel $L_{\beta^{\prime}}$ to liquid disorder $L_{\alpha}$ state. The result agrees well with previous values of $\left(n_{e}-n_{o}\right)$ in the $L_{\beta^{\prime}}$ phase and translates to an assumed average acyl chain orientation relative to the membrane normal $\left(\langle\phi\rangle \sim 32^{\circ}\right)$ when $T<T_{m}$ and $0^{\circ}$ when $T>T_{m}$. Evidence of super heating and cooling are presented. A discussion on how the observed behavior as $T_{m}$ is approached, could relate to the existence of varying microconfigurations within the lipid bilyer are presented. This conversation includes ideas from a Landau-Ginzburg picture of first-order phase transitions in nematic-to-isotropic systems.
\end{abstract}

DOI: 10.1103/PhysRevE.101.032416

\section{INTRODUCTION}

Charting the complex interface of biological membranes is arguably the most essential piece in determining how localized interactions contribute to overall cellular function. These membranes contain an elaborate mesh of membrane proteins, various lipids, and an assortment of domains. Studying the regional lipid orientation within membranes could provide us with a better insight on the interactions between neighboring molecules. Instead of approaching the complexities of a true cellular membrane, model membranes systems are often used. These model membrane structures have been characterized using techniques ranging from nuclear magnetic resonance (NMR) to various $x$-ray methods [1-5]. These routines accurately predict structural properties of lipid membrane multilayers and have given us a better understanding of the physical behavior of these systems. Techniques such as NMR and x-ray scattering are not commonly used on low curvature-single bilayer systems, which are naturally found in biological cellular membranes. In general these methodologies are limited in obtaining localized lateral information of planar membrane systems.

Many groups have chosen methods that mainly focus on exploiting the anisotropic nature of lipids to obtain structural

\footnotetext{
*johnsonm@pfw.edu

†rdecca@iupui.edu
}

information of the lipid molecules in the membrane [3,6-9]. Techniques ranging from ellipsometry [10] to surface plasmonic resonance [11] exploit the birefringent properties of lipid bilayers to study membrane structure. More specifically, various studies have used the anisotropic properties of 1,2-dipalmitoyl-sn-glycero-3-phosphocholine (DPPC) bilayers in the $L_{\beta^{\prime}}$ gel state to measure the effective retardance of the membrane system $[9,12,13]$. Under these conditions the lipid molecules are assumed to possess an average constant molecular tilt, $\phi \sim 32^{\circ}$ [14], across the structure. Knowing the perpendicular component of the refractive index $n_{\perp}$ of the acyl chains, the optical orientation $\theta$ and parallel refractive index $n_{\|}$of the acyl chains can be determined. From this information one is able to resolve changes in the properties of the membrane. All mentioned techniques were utilized in studying both bulk and planar lipid membrane systems. One common drawback is that they are all limited in the lateral information they can obtain. To address this issue Lee et al. [12] were able to explore these structural properties by measuring the effective retardance, using a near-field scanning optical microscope (NSOM). Using NSOM one is able to obtain optical information on the order of the size of the aperature of the NSOM probe. This gave Lee et al. a lateral resolution on the order of $\sim 100 \mathrm{~nm}$ across the planar membrane system. Though they were able to obtain a high lateral sensitivity using NSOM, the measurement of the retardance was precision limited, leaving room for improvement on the detectability of the acyl chain tilt variations across planar membrane systems. 
In an attempt to advance this work, Johnson et al. [15] implemented a polarization modulation (PM) technique to study topological defects across the membrane, which only slightly improved on the previous measurements conducted by Lee et al. [12].

In studying lipid systems it is also common to investigate their thermodynamic properties to better understand the localized interactions that take place between molecules. Temperature-controlled NMR and $\mathrm{x}$-ray experiments $[1,5,16-18]$, in addition to differential scanning calorimetry $[5,19,20]$ have been used to study the first-order phase transitions in lipid systems. As an example, one can study the main transition from the gel $L_{\beta^{\prime}}$ to liquid disordered $L_{\alpha}$ state in phosphocholine lipid membranes with saturated acly chains. For systems like DPPC bilayers the average orientation with respect to the normal direction of the membrane surface goes from having some characteristic tilt to having an average orientation along the normal direction. Detecting these structural changes could allow one to determine the phase transition conditions of the system.

In this paper, we will highlight an improved polarization modulation technique [21] that was combined with a temperature-controlled near-field scanning optical microscope (PM-NSOM). We will discuss how the system was calibrated and showcase how one is able to measure the relative change in the retardance $(\Delta S)$ versus an absolute retardance to improve the sensitivity of the measurement. We will also highlight how this was imperative in performing temperature-based polarization measurements on supported membrane samples. We will describe how one is able to not only obtain the average orientation of the acyl chains with respect to the membrane's normal, but also the direction of the acyl chain projection in the plane perpendicular to the membrane's normal. These attributes will be displayed using images that highlight the edge effects near defects in a planar membrane. The retardance in a supported DPPC bilayer was investigated as a function of temperature. The improvement in the apparatus yielded an enhanced precision in the retardance of a single DPPC bilayer, resulting in a more accurate difference between $n_{\perp}$ and $n_{\|}$over a lateral size of $\sim 100 \mathrm{~nm}$. Furthermore, the gel-liquid crystalline phase transition of DPPC has been investigated with the same spatial resolution. These systems were also studied as they were superheated and cooled. The results from these measurements will be discussed. We expect that the technique presented here could help with future investigations in determining the sizedependent transition temperature in mixtures of in-miscible lipids in planar bilayer systems. This will provide a new tool to investigate the intermolecular interactions between different lipids, which may address many of the previous described limitations found in other techniques $[1-3,17,18]$.

\section{EXPERIMENTAL DETAILS}

\section{A. Sample preparation}

Hydrophilic glass substrates were prepared by sonicating Fisher brand glass cover slips in detergent and deionized (DI) water separately for $\sim 15-20 \mathrm{~min}$ each. This was followed with a wet-chemical oxidation process using a piranha

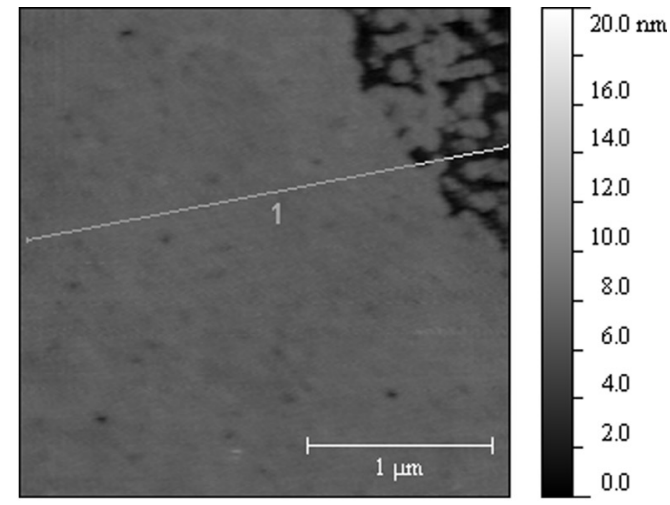

(a)

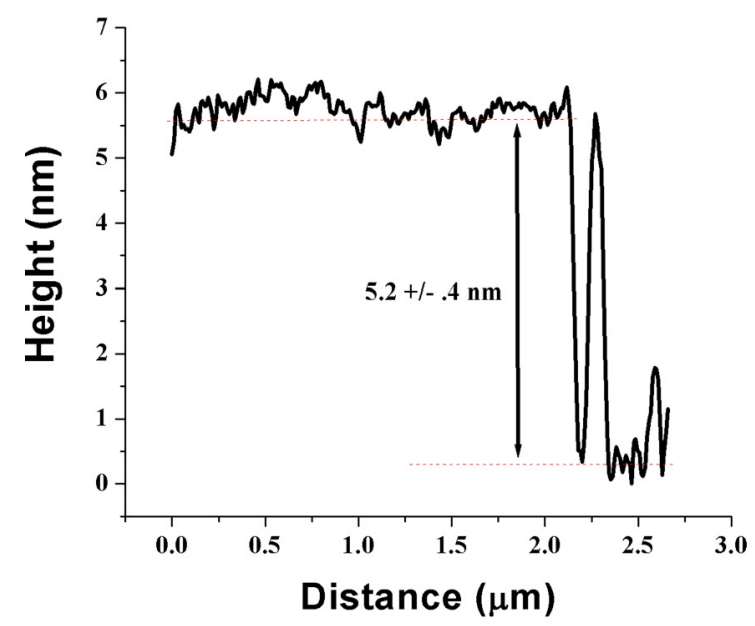

(b)

FIG. 1. AFM image of supported DPPC bilayers near an edge. (a) $2.6 \mu \mathrm{m} \times 2.6 \mu \mathrm{m}$ topographical contrast image. The low height regions represent the underlying glass slide. (b) Plot of the height data taken from a line cut 1 of the image in (a), showing a height difference of $\approx 5.2 \mathrm{~nm}$.

solution $\left(\mathrm{H}_{2} \mathrm{SO}_{4}: \mathrm{H}_{2} \mathrm{O}_{2}=3: 1\right)$ for $\approx 5 \min [22,23]$, to create a flat hydrophobic surface. The cover slips were then rinsed and sonicated for $\sim 30 \mathrm{~min}$ in DI water and finally thermally dried under normal atmospheric conditions.

Supported lipid bilayers were formed on the prepared hydrophilic glass substrates by vesicle fusion [23,24]; 1.0$0.5 \mathrm{mg} / \mathrm{ml}$ of 1,2-dipalmitoyl-sn-glycero-3-phosphocholine (DPPC) in $100 \mathrm{mM} \mathrm{NaCl}: 30 \mathrm{mM} \mathrm{NaH} \mathrm{PO}_{4}$ was sonicated at $\approx 60^{\circ} \mathrm{C}$ until clear to create small unilamellar vesicles (SUV's); $100 \mu \mathrm{L}$ of solution was placed on the glass substrates and left to equilibrate at room temperature for $\approx 30 \mathrm{~min}$. The samples were then baked at $60^{\circ} \mathrm{C}$ for $45-$ $60 \mathrm{~min}$. After rinsing the samples with DI water, they were either rehydrated and characterized with an AFM under a fluid cell (see Fig. 1), or kept in a chamber at $100 \%$ relative humidity until imaged with the PM-NSOM system.

\section{B. Experimental setup and supporting details}

NSOM probes were created by heating and pulling stripped single-mode optical fiber with a micropipette puller (SUP2000 Sutter instruments). All probes utilized in experiments 
were coated with $\sim 5 \mathrm{~nm}$ of $\mathrm{Cr}$ and $\approx 150-200 \mathrm{~nm}$ of Al. The tip diameters were determined to be $\approx 80-100 \mathrm{~nm}$ (characterized using a scanning electron microscope). All experimental probes' polarization behavior was characterized using a universal polarizer and analyzing linear polarizer. Only NSOM probes with polarization extinction ratios that were at least 100:1 were considered acceptable and utilized in the system. The total PM-NSOM setup is schematically shown in Fig. 2. Monochromatic light from a $(5-6 \mathrm{~mW})$ helium neon laser $(\lambda=632.8 \mathrm{~nm})$ was mechanically chopped and passed through a linear polarizer. All other optical elements were positioned with respect to this direction defined to be $0^{\circ}$. After the linear polarizer, light passed through a photoelastic modulator (PEM) $\left(45^{\circ}\right.$, frequency $\left.41.9 \mathrm{kHz}\right)$ and quarter wave plate (QWP) $\left(0^{\circ}\right)$. The light was then coupled into a single-mode optical fiber, which was connected to a universal fiber polarizer, used to control the polarization going into the NSOM probe [21]. The NSOM tip-to-sample distance $(\sim 10 \mathrm{~nm})$ was controlled utilizing a shear force feedback system $[25,26]$. The light from the NSOM probe and sample was collected with an objective lens, and sent through a QWP $\left(0^{\circ}\right)$, an analyzer $\left(-45^{\circ}\right)$, and finally collected by a Si PIN diode detector. The current from the diode was converted to a voltage signal by means of a transimpedance amplifier and measured by three lock-in amplifiers (LIA). The three LIAs were locked at the frequency of the mechanical chopper (proportional to the intensity of the laser, DC value), the first and second harmonics of the PEM frequency, respectively.

In the absence of a sample, it was initially assumed that the universal polarizer negated any polarizing effects from the fiber and NSOM probe. Under these circumstances, a Jones matrix formalism was applied and it was determined that the signal at the detector is proportional to $\frac{1}{2}-$ $\frac{1}{2} \sin (A) \sin (S) \cos (2 \theta)+\frac{1}{2} \cos (A) \sin (S) \sin (2 \theta)$, where $A=$ $B \cos (\omega t), B$ is the magnitude of the retardance, and $\omega$ is the angular frequency set by the PEM (The PEM was calibrated such that the Bessel function of the first kind, $J_{0}(B)=0$ ). The lock-in amplifiers are used to obtain a signal normalized by the DC term at the first harmonic $\left(I_{\omega}\right)$ and the second harmonic $\left(I_{2 \omega}\right)$,

$$
\begin{aligned}
& I_{\omega}=\gamma J_{1}(B) \sin (S) \cos (2 \theta), \\
& I_{2 \omega}=\gamma J_{2}(B) \sin (S) \sin (2 \theta),
\end{aligned}
$$

where $J_{1}(B)$ and $J_{2}(B)$ are Bessel functions of the first kind, which appear due to the sinusoidal temporal dependence on A. Hence,

$$
\theta=\frac{1}{2} \arctan \left(\frac{-I_{2 \omega} J_{1}(B)}{I_{\omega} J_{2}(B)}\right),
$$

and the retardance $S$ is

$$
\begin{aligned}
S & =\arcsin \left(\frac{I_{\omega}}{\gamma J_{1}(B) \cos (2 \theta)}\right) \\
& =\arcsin \left(\frac{-I_{2 \omega}}{\gamma J_{2}(B) \sin (2 \theta)}\right),
\end{aligned}
$$

where $\gamma$ is a the term associated with the lock-in measurement (i.e., $\gamma=\frac{\pi}{2}$ due to the DC component being a square wave for our study). During the alignment process in the absence of a

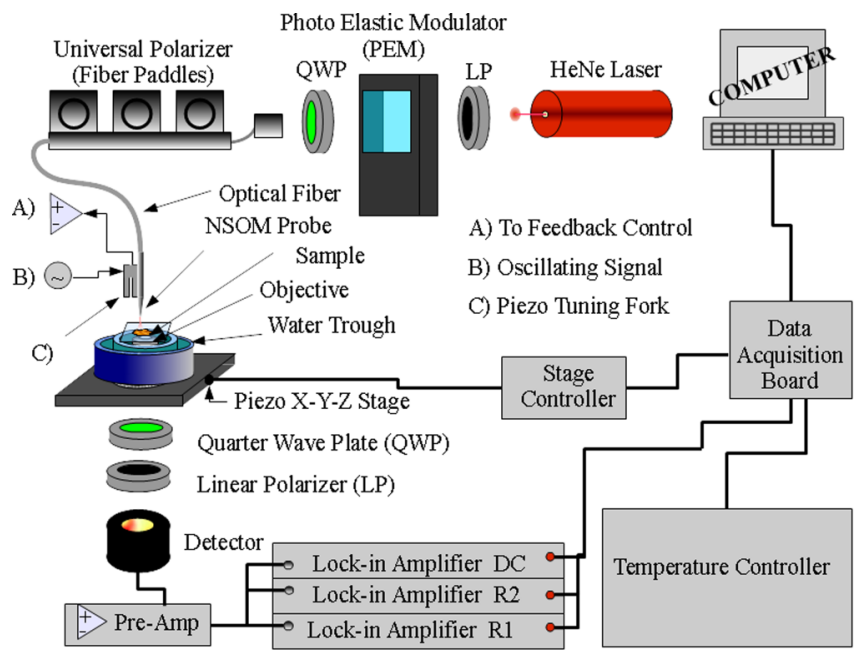

(a)

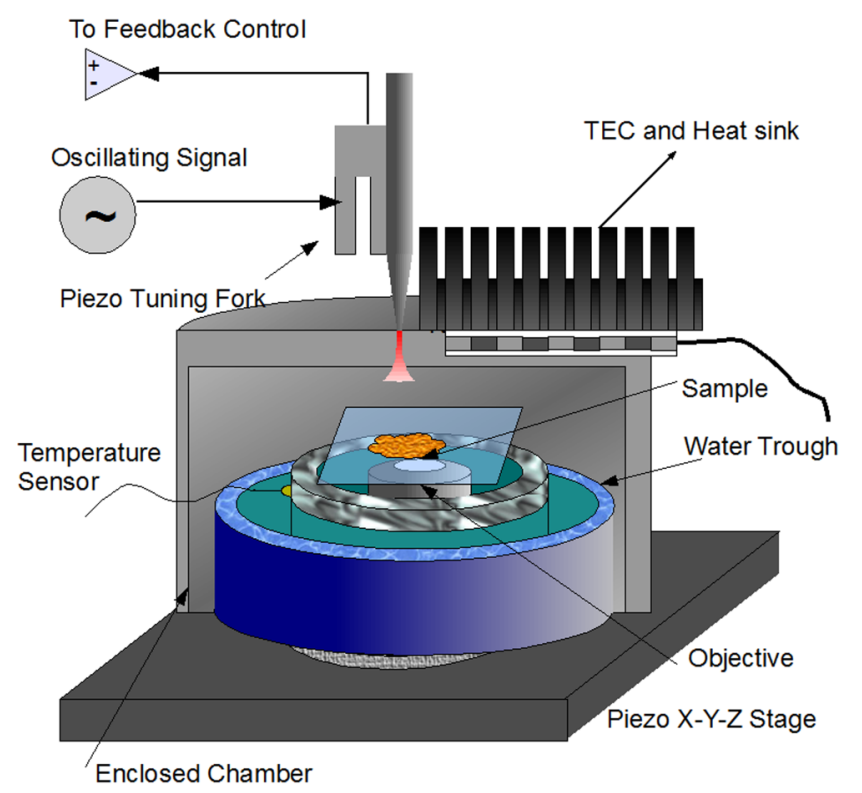

(b)

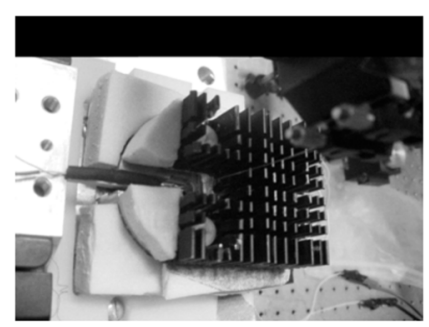

(c)

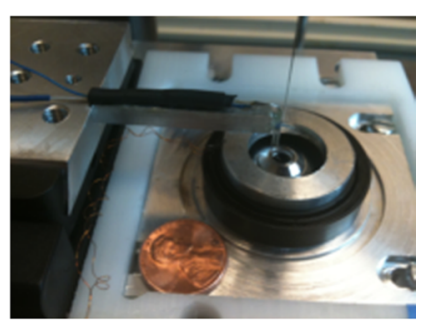

(d)
FIG. 2. Diagram of PM-NSOM setup. (a) PM-NSOM setup highlighted with components for room temperature studies (b) Diagram of the temperature-controlled chamber with water trough to maintain $100 \%$ relative humidity. (c) and (d) Photographs of the temperature-controlled chamber.

sample, both $I_{\omega}$ and $I_{2 \omega}$ in Eqs. (1) and (2) are minimized, which implies that the retardances associated with the NSOM probe and fiber approach zero. 
Moving the sample in and out of the optical path while aligning the NSOM system is not ideal due to the user's risk of damaging the probe and the cumbersome process of attaching a replacement. It would be advantageous to have a method where the alignment could be made over the sample of unknown retardance. To understand this effect, the sample and probe were modeled as two independent retarding elements, with retardances $S$ and $\delta$ inserted between the two QWP in the previously described setup. Following the same Jones matrix formalism the expected intensities of the two retarding objects $S$ and $\delta$ oriented at different angles were calculated. During the alignment process, where $I_{\omega}$ and $I_{2 \omega}$ are minimized, we can assume the orientation of the NSOM probe/fiber and sample are equivalent. Using the assumption that the changes in retardances measured were relativly small in magnitude, the intensities,

$$
I_{\omega}=\gamma J_{1}(B) \sin (S+\delta) \cos (2 \theta)
$$

and

$$
I_{2 \omega}=\gamma J_{2}(B) \sin (S+\delta) \sin (2 \theta),
$$

are obtained. From these expressions, it is seen that instead of an absolute retardance value, the result is the sum of the two retarding objects. Therefore, one is able to align the system over the sample using the universal polarizer, making $S+\delta \rightarrow 0$, and obtain a deviation in the retardance $\Delta S$ and a relative orientation $\theta$ across a given sample.

To ensure the correctness of our calibration approach and the viability of our previous calculations, PM-NSOM measurements were conducted on cleaved stepped muscovite (mica) substrates. The mica substrate showed steps of different heights corresponding to a different number of layers of the crystal. Both far-field and near-field measurements were made of the retardance $S$ on mica; the far-field measurements were used to make sure NSOM determinations were accurate. Both aforementioned approaches for measuring the retardance and orientation using the NSOM apparatus were performed. It was observed that on average the overall difference was $\Delta S \sim 0.3 \mathrm{mrad}$ between the two methods; see Fig. 3. Using the height topography from the NSOM measurement the birefringence of the muscovite crystal was determined to be $\sim 0.0025$, which falls within accepted values $[27,28]$. It was noted that the images taken with the system aligned over the sample yielded more detail in $\Delta S$ than just measuring $S$. We suspect that this increase in sensitivity to sample variations is a consequence of the measurements being done from a minimum instead of an offset signal, highlighting an additional advantage of aligning the system over the sample.

All of the supported lipid bilayer experiments were conducted at $100 \%$ relative humidity, to ensure membrane structure contained the characteristic acyl chain tilt of $\approx 32^{\circ}$ in the $L_{\beta^{\prime}}$ phase at room temperature. The measurements were taken with scan times ranging from 100 to $450 \mathrm{~ms}$ per point, where $I_{1 \omega}, I_{2 \omega}$, and topography data were collected using a data acquisition board and LABVIEW computer program. Trace and retrace images were collected to confirm measurement reliability.

Temperature-controlled PM-NSOM measurements were performed similarly to those conducted at room temperature, but included the following procedures. A cylindrical

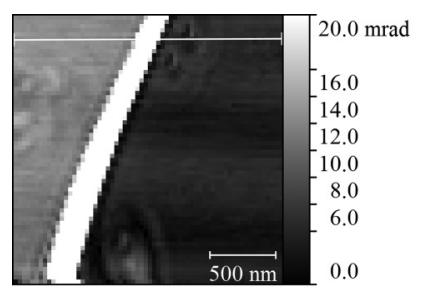

(a)

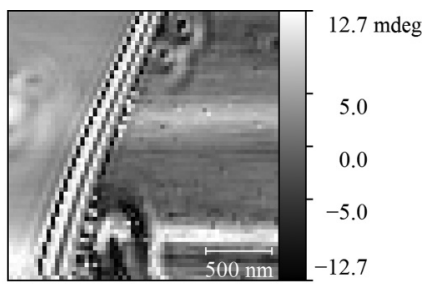

(b)

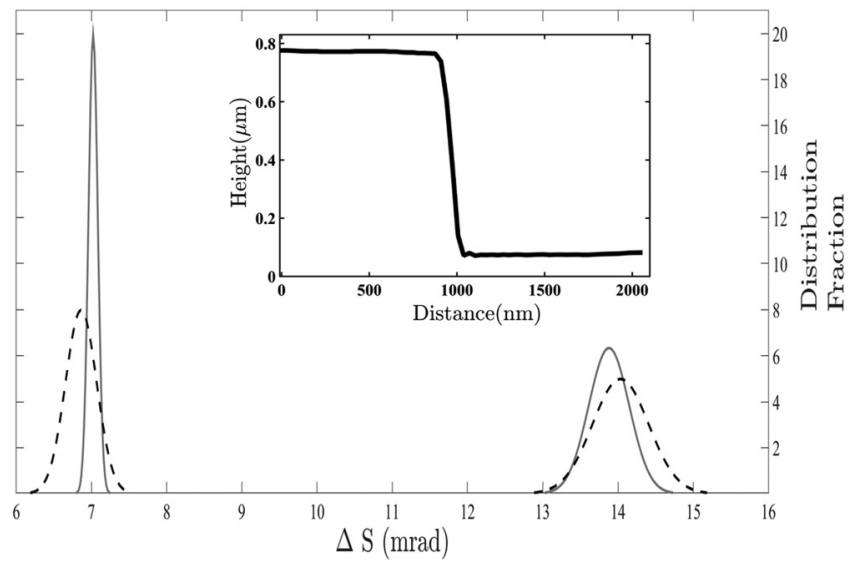

(c)

FIG. 3. PM-NSOM picture of muscovite. (a) $\Delta S$ as a function of position with the system aligned over the sample. (b) $\theta$ as a function of position with the system aligned over the sample. (c) Graph of the distributions of the values of $S$ and $\Delta S$ at different regions of the muscovite crystal. Histograms of $S$ and $\Delta S$ values were made, where Gaussian curves were fit to the peaks of the results. (Dashed line) Measurements of the sample where the system was not aligned over the sample $(S)$. (Solid gray line) Measurements of the sample where the system was aligned over the sample $(\Delta S)$. (Inset) Height as a function of position of the line shown in (a).

aluminum chamber with a narrow slit in one side for accommodating the NSOM probe holder was placed around the NSOM probe (Fig. 2) to minimize the lateral heat gradients across the sample. The chamber was thermally isolated with an insulating foam casing and the temperature was controlled using a Peltier thermoelectric cooler (TEC) to move thermal energy in and out of the chamber. The temperature of the sample was measured using a calibrated thermistor embedded into the aluminum sample holder. Thermistors were calibrated utilizing boiling points of deionized water, ethanol, isopropanol, methanol, and acetone. The sample holder rested on a Delrin stand with a stainless steel bottom to improve mechanical stability. With this design the temperature stability was within $\pm 0.07^{\circ} \mathrm{C}$. A water trough surrounded the sample holder, which preserved the required $100 \%$ relative humidity.

During a typical run the chamber's temperature was first heated beyond $T_{m}$ to ensure the lipid membrane was well into the $L_{\alpha}$ state. The NSOM probe was then engaged with the sample. The chamber was cooled below $T_{m}$ and heated back to the initial temperature. This was proven to be the most reliable method to counter the probe crashing events that occurred due to the thermal expansion of the Delrin separator. 


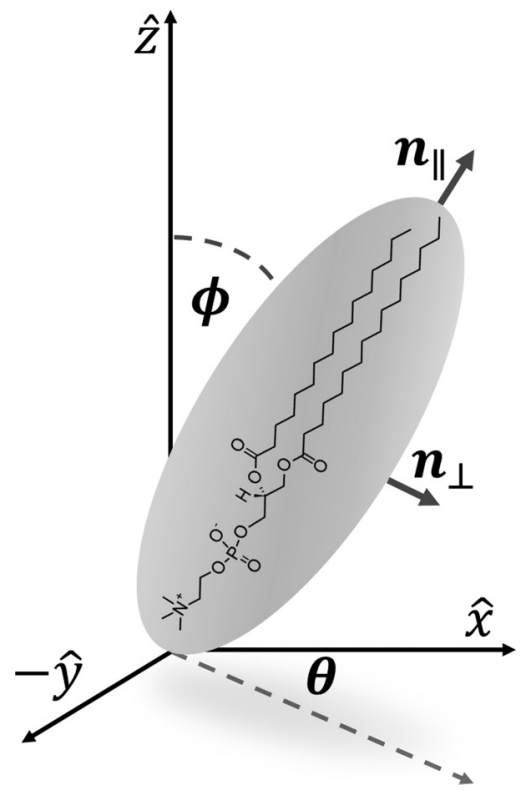

FIG. 4. Pictorial representation of the lipid molecule and its indicatrix. The image highlights how the molecule orientation is described in terms of $\theta$, optical orientation $(\phi)$, and perpendicular and parallel indices of refraction.

\section{RESULTS}

The anisotropic nature of lipids yields differences in the index of refraction [or birefringence $\left(n_{e}-n_{o}\right)$ ] along orthogonal directions in the plane of the membrane's surface. Because the polarizability of the lipid molecule is asymmetric, the refractive indices along the length of the acyl chains $\left(n_{\|}\right)$and perpendicular $\left(n_{\perp}\right)$ are not equal. In this study, the principal optical axis is assumed to lie parallel to the length of the acyl chains, $\phi \sim 32^{\circ}$ with respect to the membranes' normal for DPPC; see a representation of the lipid molecule in Fig. 4. For all calculations, $n_{\|}$was assumed to be 1.4 [12]. Polarized light of wavelength $\lambda$ propagating in the $z$ direction, parallel to the membranes' normal, experiences a retardance between the $x$ and $y$ components of the electric field according to [12]

$$
\begin{aligned}
\Delta S= & \frac{2 \pi\left(n_{e}-n_{o}\right) t}{\lambda} \\
= & \frac{2 \pi t}{\lambda} \frac{1}{\sin \left(\arctan \left(\frac{n_{\|}^{2}}{n_{\perp}^{2}} \cot (\phi)\right)+\phi\right)} \\
& \times \frac{n_{\perp} n_{\|}}{\sqrt{n_{\perp}^{2} \sin ^{2}(\phi)+n_{\|}^{2} \cos ^{2}(\phi)}}-n_{\perp} .
\end{aligned}
$$

The extraordinary ray corresponds to the electric field parallel to the optical axis, where the electric field direction for the ordinary ray is perpendicular to that axis. Due to the small thickness of the membrane, the two rays were indistinguishable with the collection optics used.

Various images were collected from different areas of the supported DPPC bilayer on glass until a discontinuity in $\Delta S$ and $\theta$ were found. From the image of $\Delta S$ in Fig. 5, a measured change in $\Delta S$ of $\approx(3.9 \pm 0.4 \mathrm{mrad})$ was obtained, between a region with no membrane and membrane. This was done by taking a line cut of the image and averaging $\Delta S$ inside the

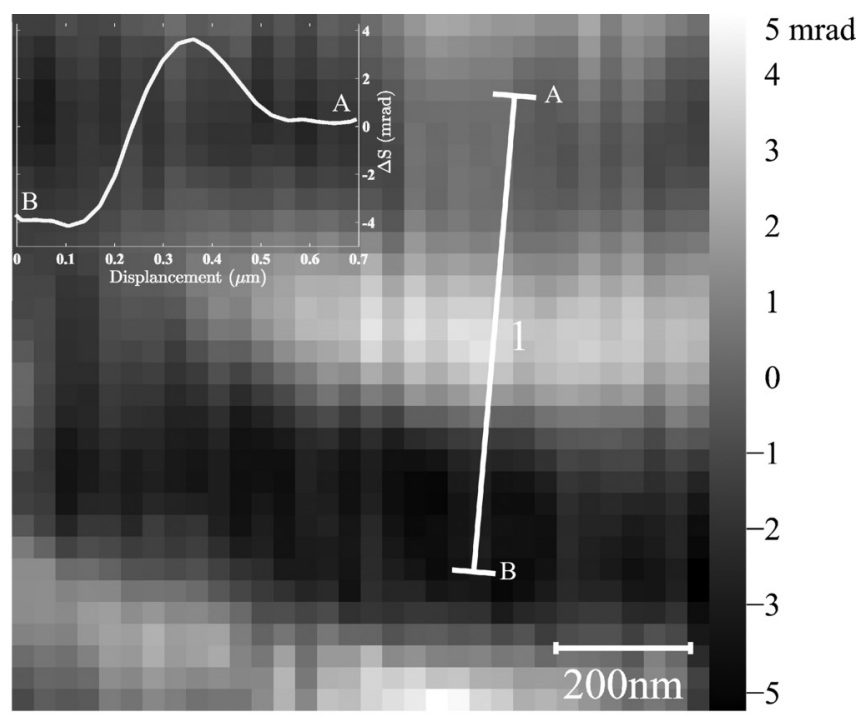

(a)

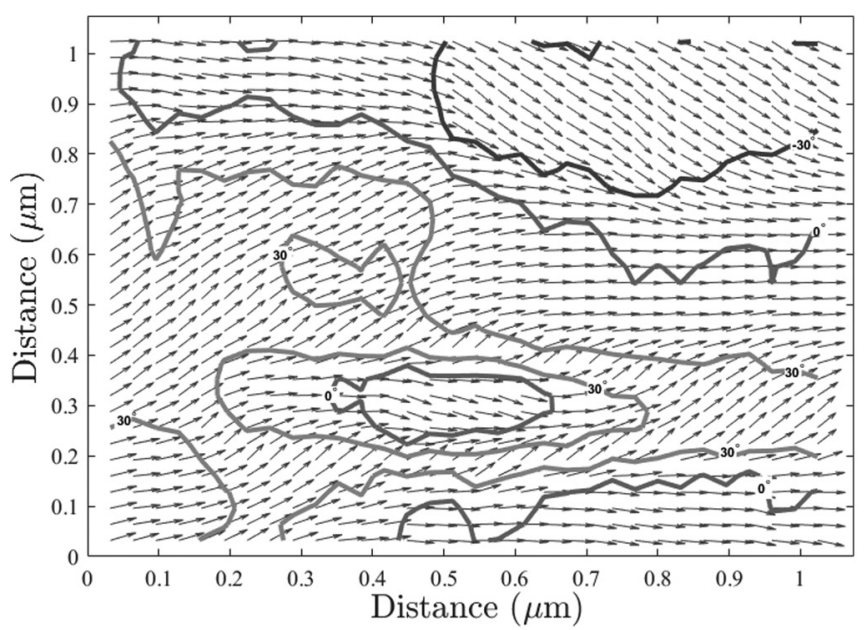

(b)

FIG. 5. PM-NSOM picture of DPPC supported on glass. (a) $\Delta S$ as a function of position. (Inset) Data taken from the line cut shown on the image. (b) Quiver-contour map of $\theta$. (Note that the angle of the quivers was increased by a factor of 5 to enhance the changes in $\theta$ across the sample.)

hole and subtracting from a region away from the determined edge. $\theta$ is plotted using a contour plot along with quivers to show the relative projection of the acyl chains. The angle of the quivers were multiplied by a factor of five to enhance the changes detected across the sample. The contour plot highlights the boundaries of the areas with similar values. The hole was easily observed using the contour plot of $\theta \cdot \theta$ inside the hole is defined by the direction of the system's axis. The birefringence was determined to be $\left(n_{e}-n_{o}\right)=\frac{\Delta S \lambda}{2 \pi t} \approx$ $0.073 \pm 0.008$, using $\lambda=632.8 \mathrm{~nm}$ and $t=(5.2 \pm 0.4 \mathrm{~nm})$ [29]. These results agree well with previous measurements with a significant reduction in error [12]. Furthermore an increase in $\Delta S$ to $\approx 7.5 \mathrm{mrad}$ was obtained at the edge of the hole. It is expected that $\phi$ is greater where the membrane forms a boundary. The topography data did not show any 
change in height, which is expected since the hole is much smaller than the tip diameter, $\approx 500 \mathrm{~nm}$ (data is not shown).

A series of temperature PM-NSOM experiments (Fig. 6) probed the main phase transition temperature $T_{m} \sim 41^{\circ} \mathrm{C}$ of DPPC across a $512 \mathrm{~nm} \times 512 \mathrm{~nm}$ area. The $16 \times 16$ pixel raster scanned images were taken over $\approx 3.5 \mathrm{~min}$, heating at a rate of $\sim 0.06{ }^{\circ} \mathrm{C} / \mathrm{min}$. The temperature is changing as a function of time. Therefore, each pixel represents a slightly different temperature change $\sim 0.8 \mathrm{mK}$. We assume the temperature to be constant over the pixel acquisition time $(\approx 0.8 \mathrm{~s})$ due to our sensitivity limits in temperature. The full series started at $T \approx 38^{\circ}$ and finished at $T \approx 42^{\circ}$. Figure 6 highlights the main transition ocurring over a single image in the series. In Fig. 6(a), $\Delta S$ is observed to remain constant at low $T$ and then shows a jump of $\Delta S \approx(3.84 \pm 0.20) \mathrm{mrad}$ at $\approx 41.1^{\circ} \mathrm{C}$. This change in $\Delta S$ is interpreted as the average position of the acyl chains change from their characteristic $\langle\phi\rangle$ of $\approx 32^{\circ}$ in the $L_{\beta^{\prime}}$ to the $\langle\phi\rangle \rightarrow 0$ in the $L_{\alpha}$ state. A change of $\approx 21^{\circ}$ at $T_{m}$ was observed in $\theta$ at the same position $\Delta S$ transitioned at in Fig. 6, $(\theta$ data not displayed). This change in $\theta$ corresponds to the sample in the $L_{\beta^{\prime}}$ state with the optical system having one $\theta$, and changing to a different value, characteristic of the optical system, when $T>T_{m}$.

Using the model previously presented and the data in Fig. 6(a) we created a three-dimensional representation of the average direction of the orientation of the acyl chains of the lipid molecules within the membrane; see Fig. 6(c). Using the values for $n_{\perp}$ and $n_{\|}$, the average orientation $\phi$ of the acyl chains across the NSOM probe's aperture with respect to the membrane's normal, was calculated. As shown, when the membrane is below $T_{m},\langle\phi\rangle$ was $\approx 32^{\circ}$ while when it is heated above the phase transition temperature it was in the $L_{\alpha}$ phase, where $\langle\phi\rangle$ is zero.

The next series of temperature-controlled experiments decreased the image acquisition time to $\approx 50 \mathrm{~s}$, the rate of temperature change was $\sim 5^{\circ} \mathrm{C} / \mathrm{h}$ over the entire scan. This was done to explore the hysteresis effects of the first-order phase transition of the planar membrane system. Images taken were identical in size to the previous described temperaturecontrolled experiments. The average $\Delta S$ of each image was taken and plotted as a function of temperature, with the error bar representing the variation across the image at that temperature. The results for $\Delta S$ are seen in Fig. 7, where a change in $\Delta S$ of $\approx 3.5 \mathrm{mrad}$ was observed across $T_{m}$. Figure 7 (a) shows that $S \approx 4.7 \mathrm{mrad}$ for $T<T_{m}$ and $\approx 1 \operatorname{mrad}$ when $T>T_{m}$. This translates to the acyl chains $\langle\phi\rangle$ transitioning from $\sim 32^{\circ}$ to zero, calculated using Eq. (7), as is expected to occur when the lipid bilayer goes from the $L_{\beta^{\prime}}$ into the $L_{\alpha}$ phase. We do note that in our measurements when $T<T_{M}$ that bilayer should exhibit properties of the ripple phase. However, due to the ripple period being smaller than the aperture of the NSOM probe, we are unable to report on the phenomena [30,31].

\section{DISCUSSION}

With the knowledge obtained from the PM-NSOM experiments at room temperature on supported DPPC in the $L_{\beta^{\prime}}$ phase, physical parameters about the lipid molecules in the membrane are extracted. Using the birefringence we obtain information on the polarizability of the lipid molecules. Fol-

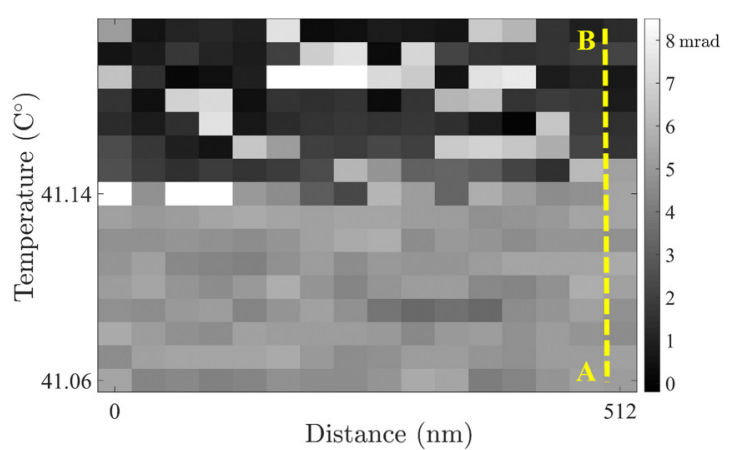

(a)

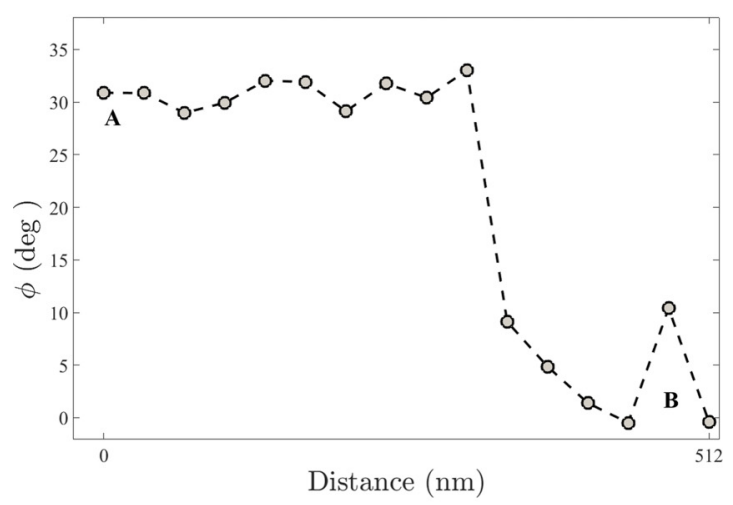

(b)

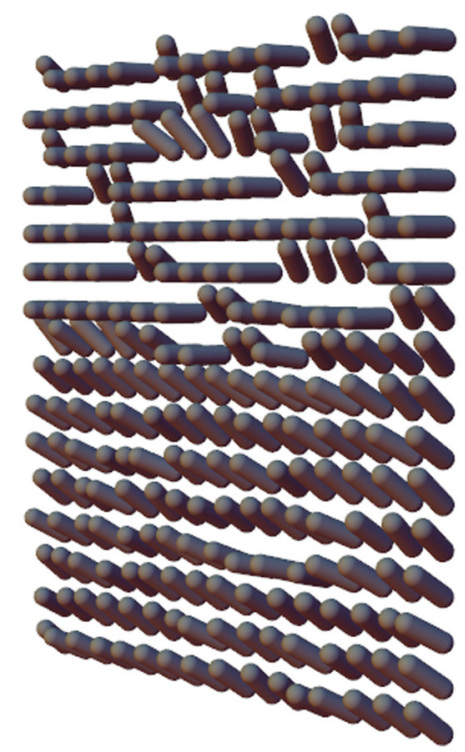

(c)

FIG. 6. (a) Temperature-controlled PM-NSOM $512 \mathrm{~nm} \times 512 \mathrm{~nm}$ images of DPPC supported on glass. The images were raster scanned, starting from the bottom right corner of the image, with an $\approx 0.8$-s pixel acquisition time. We assumed a homogeneous temperature over the acquisition time for each pixel. An image of the measured $\Delta S$, showing a change in $\Delta S$ of $\approx 3.8 \pm 0.3 \mathrm{mrad}$ at the $T_{m}$. This change in $\Delta S$ indicates a change of $\phi$ of the acyl chains. The temperature scale on the left side highlights the initial temperature of the first pixel and the temperature where we observed a transition in $\Delta S$. (b) Graph of the calculated $\phi$ as a function of position using data taken in the region of the dashed line in (a). (c) Three-dimensional representation of the average acyl chain orientation throughout the membrane. Each tube portrays the average position of many lipid molecules contained in an $\sim(100 \mathrm{~nm})^{2}$ area. 


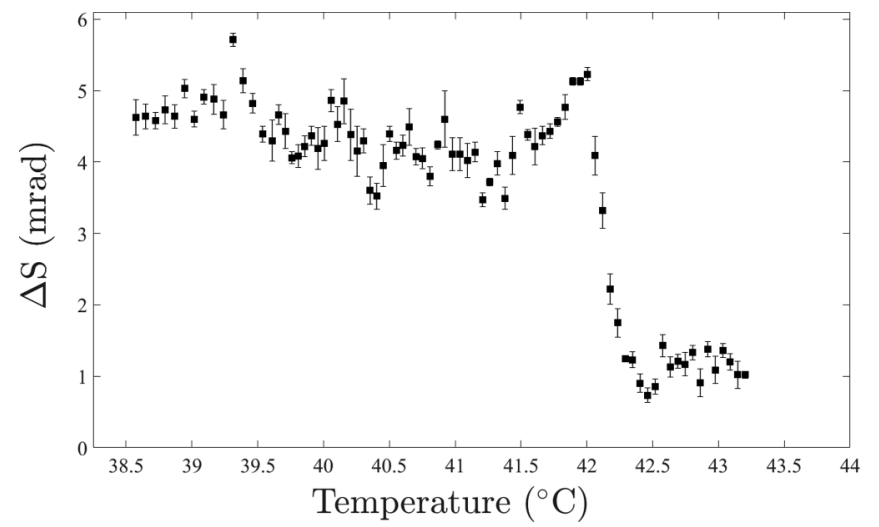

(a)

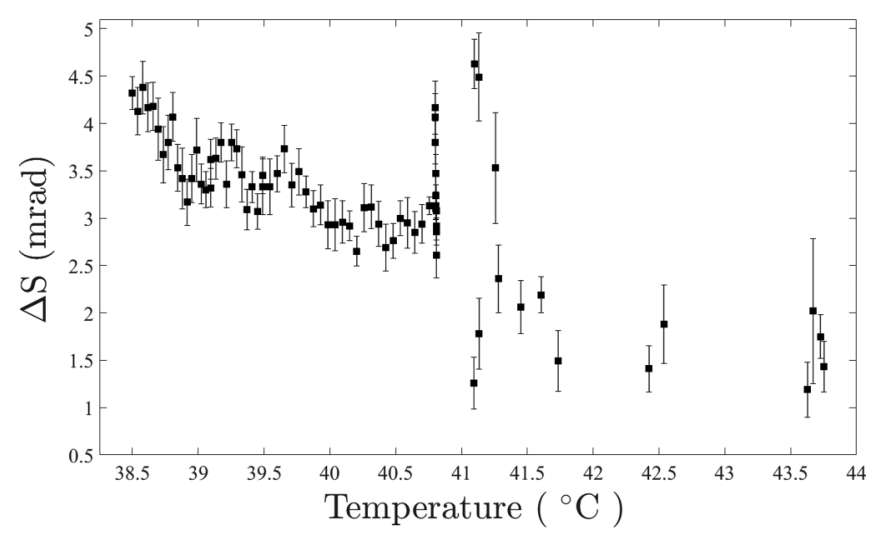

(b)

FIG. 7. $\Delta S$ values from a temperature-controlled PM-NSOM measurement of DPPC supported on glass. (a) Graph of $\Delta S_{\text {avg }}$ versus temperature as the system was heated, taken from $(512 \mathrm{~nm})^{2}$ images showing a change in $\Delta S$ across $T_{m}$ of $\approx 4.0 \pm 0.4 \mathrm{mrad}$. (b) Cooling data graph of $\Delta S_{\text {avg }}$ versus temperature, taken from $\sim(100 \mathrm{~nm})^{2}$ images.

lowing the model of rigid cylinders used by Salamon et al. [9] Eq. (7) and the relationship $n_{i}{ }^{2}=\frac{\alpha_{i} \epsilon_{o}}{\left(V-\alpha_{i} L_{i}\right)}+\epsilon_{o}$ (where $V$ is volume and $L_{i}$ is the shape factor for a cylinder [32]) we were able to determine the transverse $\left(\alpha_{t}\right)$ and longitudinal $\left(\alpha_{l}\right)$ polarizabilites. The values for the two acyl chains were determined to be $\alpha_{t}=44.2 \AA^{3}$ and $\alpha_{l}=94.4 \AA^{3}$ assuming the area per lipid $(A)$ and acyl chain length $(l)$ to be $47.9 \AA^{2}$ and $17.2 \AA$, respectively [14]. These values are very close to the theoretically calculated polarizabilities $\alpha_{t}=25.14 \AA^{3}$ and $\alpha_{l}=45.8 \AA^{3}[9,32]$ of a single palmitic acid $\mathrm{C}_{16}$.

The hysteresis observed under the applied heating rates are comparable to what has previously been reported $[19,33]$. To investigate the localized homogeneity of the sample in Fig. 7(a), the variance $\left(\sigma_{\Delta S}^{2}\right)$ of a $150 \mathrm{~nm} \times 150 \mathrm{~nm}$ region was analyzed. From the results, we observed that $\langle\Delta S\rangle$ fluctuated as $T_{m}$ was approached; see Fig. 7. This phenomena is further highlighted in the graph of the variance versus temperature; see Fig. 8(b). We can attempt to explain these fluctuations using two avenues of thought. One could derive from the observation of varying microconfigurations that increase in number as $T_{m}$ is approached, as predicted by previous computational work on lipid phase transitions [34,35]. Unfortunately, the

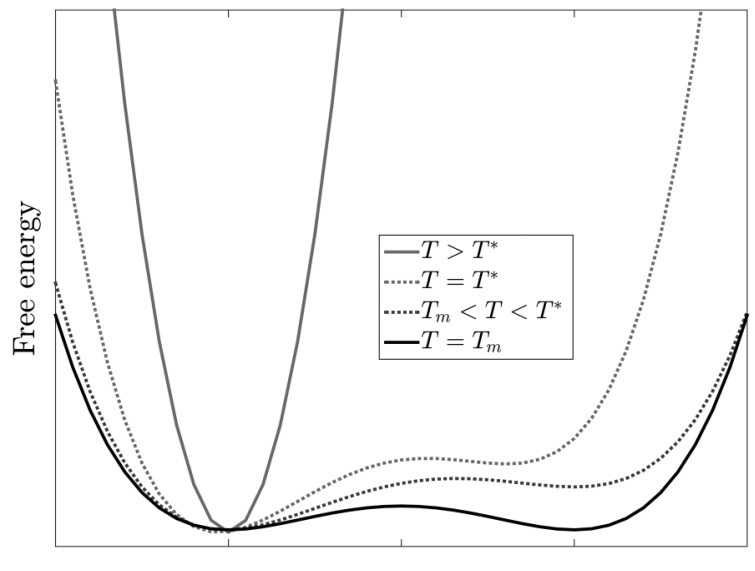

$\Gamma$

(a)

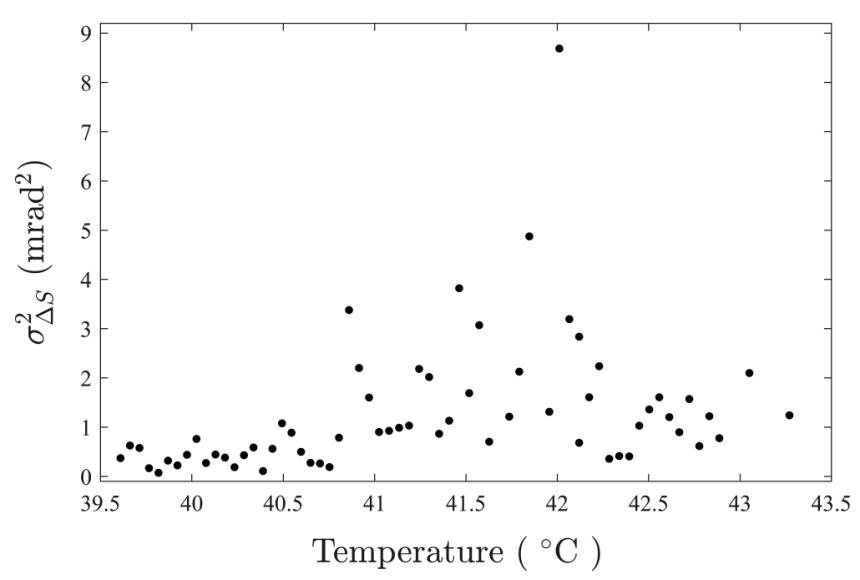

(b)

FIG. 8. (a) Graphical depiction of the free energy of a system as a function of the order parameter( $\Gamma$ ). (b) Graph of $\sigma_{\Delta S}^{2}$ versus $T$, created by analyzing the heating data presented in Fig. 7(a).

time scale of our measurement compounded by the probe size does not allow us to confirm these microconfiguration fluctuations with PM-NSOM. It only allows us the opportunity to speculate from the current results. The other utilizes a Landau-Ginzburg picture of the first-order phase transition in nematic-to-isotropic systems [36]. It is expected that as the system crosses the melting temperature $\left(T_{m}\right)$ and approaches the super-cooled or super-heated temperature $\left(T^{*}\right)$ the shape of the free energy curve changes, as shown in Fig. 8(a), where the free energy is depicted as a function of the order parameter $(\Gamma)$. In the process of heating, where $T$ is beyond $T_{m}$, the free energy functional shape and width of the metastable minimum slightly increases, allowing for an increase in the fluctuations of $\Gamma$ and consequently of any thermodynamic quantity. The deviations from the mean in $\Gamma$ increase and therefore can be correlated to the subtle growth in the value of $\sigma_{\Delta S}^{2}$ as the system approached $T^{*}$. The increase in the variance in $\Delta S$ correlates with an oscillation in state variability from Fig. 8(a). It can be clearly seen that before the system reaches $T_{m}, \sigma_{\Delta S}^{2}$ is smaller in magnitude than when the system approaches $T^{*}$ $\left(\sim 42^{\circ} \mathrm{C}\right)$. This may suggest that this observation highlights 
these fluctuations in the metastable region predicted in the Landau-Ginzburg model, which will be further investigated in future work.

\section{CONCLUSIONS}

PM-NSOM was utilized in determining the anisotropic structural properties in supported lipid bilayers. The technique was shown to improve previous measurements on $S$ by measuring $\Delta S$ and independently determining the direction of the projection of the acyl chains onto the membrane's surface. It was also shown that one can obtain accurate lateral high resolution $\Delta S$ and orientation images with the PM-NSOM system while aligning the system over the sample. With that increased sensitivity, the longitudinal and transverse polarizability were more accurately determined yielding results comparable to their theoretically calculated values. In addition, having this sensitivity proved to be imperative in conducting the temperature-controlled experiments and allowed one to measure relative values of $\Delta S$ and $\theta$.

By adding temperature control to PM-NSOM the main phase transition from the gel state to the liquid disorder phase was observed in a planar supported bilayer with a lateral resolution $\sim 100 \mathrm{~nm}$. The melting temperature of a single DPPC lipid bilayer was found to be in good agreement with previously reported work. The information from $\Delta S$ and $\theta$ allowed for the creation of a three-dimensional model of the average orientation of the lipid molecules within the membrane. The system's sensitivity and control allowed for the observations of increased fluctuations as the metastable region of the phase diagram of the lipid membrane was probed. Future work could extend into membrane systems containing lipid mixtures or even protein-lipid complexes, where the properties of domains with different phases or orientations may be explored. With temperature control, the lateral dynamics of phase separations or even changes in the orientation of the lipid bilayer through regions of interest could be observed. Overall it was shown how PM-NSOM can be utilized as a highly sensitive and noninvasive technique to study single lipid bilayer systems and achieve structural information beyond the ability of conventional optical techniques.

\section{ACKNOWLEDGMENTS}

We would like to acknowledge the use of the atomic force microscope associated with the Integrated Nanosystems Development Institute (INDI) and Nanoscale Imaging Center (NIC) at Indiana University - Purdue University Indianapolis.
[1] J. Davis, Biophys. J. 27, 339 (1979).

[2] N. Kučerka, Y. Liu, N. Chu, H. I. Petrache, S. Tristram-Nagle, and J. F. Nagle, Biophys. J. 88, 2626 (2005).

[3] S. Tristram-Nagle, R. Zhang, R. M. Suter, C. R. Worthington, W. Sun, and J. F. Nagle, Biophys. J. 64, 1097 (1993).

[4] J. F. Nagle, K. Akabori, B. W. Treece, and S. Tristram-Nagle, Soft Matter 12, 1884 (2016).

[5] G. Neunert, J. Tomaszewska-Gras, P. Siejak, Z. Pietralik, M. Kozak, and K. Polewski, Chem. Phys. Lipids 216, 104 (2018).

[6] C. D'Ambriosio and L. Powers, Biophys. J. 27, 15 (1979).

[7] K. Mishima, K. Satoh, and T. Ogihara, Biochim. Biophys. Acta 898, 231 (1987).

[8] A. Mashaghi, M. Swann, J. Popplewell, M. Textor, and E. Reimhult, Anal. Chem. 80, 3666 (2008).

[9] Z. Salamon and G. Tollin, Biophys. J. 80, 1557 (2001).

[10] C. M. González, G. Pizarro-Guerra, F. Droguett, and M. Sarabia, Biochim. Biophys. Acta 1848, 2295 (2015).

[11] P. Parkkila, M. Elderdfi, A. Bunker, and T. Viitala, Langmuir 34, 1881 (2018).

[12] C. W. Lee, R. S. Decca, S. R. Wassall, and J. J. Breen, Phys. Rev. E 67, 061914 (2003).

[13] A. Erbe and R. Sigel, Eur. Phys. J. E 22, 303 (2007).

[14] J. F. Nagle and S. Tristram-Nagle, Biochim. Biophys. Acta 1469, 159 (2000).

[15] M. Johnson and R. Decca, Opt. Commun. 281, 1870 (2008).

[16] A. Keyvanloo, M. Shaghaghi, M. J. Zuckermann, and J. L. Thewalt, Biophys. J. 114, 1344 (2018).

[17] H. Reinl, T. Brumm, and T. M. Bayerl, Biophys. J. 61, 1025 (1992).

[18] J. Eisenblätter and R. Winter, Biophys. J. 90, 956 (2006).

[19] R. N. A. H. Lewis, N. Mak, and R. N. McElhaney, Biochemistry 26, 6118 (1987).
[20] M. Mamusa, A. Salvatore, and D. Berti, Langmuir 34, 8952 (2018).

[21] A. L. Campillo and J. W. P. Hsu, J. Appl. Phys. 91, 646 (2002).

[22] H. Okorn-Schmidt, IBM J. Res. Dev. 43, 351 (1999).

[23] K. J. Seu, A. P. Pandey, F. Haque, E. A. Proctor, A. E. Ribbe, and J. S. Hovis, Biophys. J. 92, 2445 (2007).

[24] P. S. Cremer and S. G. Boxer, J. Phys. Chem. B 103, 2554 (1999).

[25] E. Betzig, P. L. Finn, and J. S. Weiner, Appl. Phys. Lett. 60, 2484 (1992).

[26] K. Hsu and L. A. Gheber, Rev. Sci. Instrum. 70, 3609 (1999).

[27] M. D. Dyar, M. E. Gunter, and D. Tasa, Mineralogy and Optical Mineralogy (Mineralogical Society of America, Chantilly, 2008).

[28] J. W. Anthony, R. A. Bideaux, K. W. Bladh, and M. C. Nichols, Handbook of Mineralogy (Mineral Data Publishing, Tucson, 2001).

[29] W. J. Sun, R. M. Suter, M. A. Knewtson, C. R. Worthington, S. Tristram-Nagle, R. Zhang, and J. F. Nagle, Phys. Rev. E 49, 4665 (1994).

[30] T. Kaasgaard, C. Leidy, J. H. Crowe, O. G. Mouritsen, and K. Jørgensen, Biophys. J. 85, 350 (2003).

[31] A. Alessandrini and P. Facci, Soft Matter 10, 7145 (2014).

[32] D. den Engelsen, Surf. Sci. 56, 272 (1976).

[33] R. Koynova, A. Koumanov, and B. Tenchov, Biochim. Biophys. Acta 1285, 101 (1996).

[34] J. H. Ipsen, K. Jørgensen, and O. G. Mouritsen, Biophys. J. 58, 1099 (1990).

[35] O. Mouritsen, Chem. Phys. Lipids 57, 179 (1991).

[36] P. Chaikin and T. Lubensky, Principles of Condensed Matter Physics (Cambridge University Press, Cambridge, 2000). 